\title{
Manifestation de la latence en ancien français aux Xème et Xlème siècles : liaison et redoublement syntaxique
}

\author{
Nathalie Chasle \\ Université Bordeaux3-UMR 5610 \\ nathalie.chasle@etu.u-bordeaux3.fr
}

\section{Introduction}

La réalisation de consonnes après les prépositions et conjonctions au début de l'ancien français présente des variations inattendues. Précisément, trois types d'alternances sont observables dans des séquences Mot1Mot2 :

$$
\begin{aligned}
& \mathrm{a}+\text { celad } \rightarrow \text { a celad (« en cachette } », \text { La chanson de Sainte Foi, 68) } \\
& \mathrm{a}+\text { ella } \rightarrow \text { ad ella ( } \text { ( à elle », ibid., 445) } \\
& \mathrm{a}+\text { lui } \rightarrow \text { a llui ( } \text { ( à lui », ibid., 348) }
\end{aligned}
$$

Au regard de phénomènes actuels, (1) et (2) semblent s'assimiler à la liaison propre au français moderne, à savoir la réalisation d'une consonne finale, normalement muette dans le mot pris isolément, en contexte \# V (soit une alternance de type [V\#] vs [VC\#], selon l'initiale du mot suivant), alors que (3) se rapproche du redoublement syntaxique reconnu en italien, qui consiste en une gémination de la consonne initiale de $\operatorname{Mot} 2([\mathrm{V \# CC}])$

Ces deux manifestations phonologiques ont connu un grand nombre d'explications théoriques qui reposent pour la plupart sur le concept de la latence. L'idée étant que les consonnes latentes se distinguent des consonnes fixes dans la mesure où elles se réalisent selon des conditions syntaxiques (Mot1 et Mot2 doivent appartenir au même syntagme) et phonologiques strictes.

Le but de cette étude est d'analyser ces consonnes spécifiques en ancien français, en partant de l'hypothèse de l'existence d'une consonne latente à la finale de Mot1, celle-ci étant justifiée par la réapparition d'une consonne finale latine anciennement prononcée puis amuïe $(a<a d)$, à l'intérieur de certains syntagmes (cas (2) et (3)).

Cette communication proposera ainsi un examen détaillé des diverses réalisations de ces consonnes, sous la forme $[\mathrm{VC} \# \mathrm{~V}]$ puis $[\mathrm{V} \# \mathrm{CC}]$, issus de quelques textes médiévaux datant du $\mathrm{X}^{\mathrm{ème}}$ et $\mathrm{XI}^{\mathrm{e} m e}$ siècles. La période de production des $\mathrm{Cl}$ étudiée étant relativement restreinte, cinq textes m'ont permis d'étayer mes corpus de latence consonantique. Ces recherches philologiques ont été effectuées en me basant directement sur ces textes ainsi qu'à l'aide du corpus de la BFM (Base de Français Médiéval, Lyon : UMR ICAR / ENS-LSH, <http://bfm.ens-lsh.fr>). Il s'agit des textes suivants, tous étant d'auteurs anonymes :

Saint Léger (STLeg), datant de la seconde moitié du $\mathrm{X}^{\mathrm{ème}}$ siècle (Avalle, 2002; Linskill, 1974).

La Vie de Saint Alexis (STAl), écrit au XI ${ }^{\text {ème }}$ siècle (Paris, 1933 ; Storey, 1968).

Boèce, texte du $\mathrm{X}^{\mathrm{ème}}$ siècle (Lavaud \& Machicot, 1950).

La passion de Clermont-Ferrand $(P C l)$, qui date de la deuxième moitié du $\mathrm{X}^{\text {ème }}$ siècle (Avalle, 2002).

La chanson de Sainte Foi (d'Agen), (ChSF), poème du XI ème siècle (Thomas, 1974).

Sur la base des données fournies, une approche théorique sera alors proposée quant aux consonnes latentes en contexte _ \# V et _ \# C, ce qui, a posteriori, permettra précisément de vérifier l'état de 
latence présupposé des consonnes finales. La théorie sera formulée selon un concept récursif de la syllabe et le postulat de la présence d'accent secondaire à la période d'ancien français étudiée.

\section{Réalisation de la consonne latente sous la forme $\mathrm{V \# CI} \mathrm{CV}$}

\subsection{Séquences $\mathrm{V} \# \mathrm{C}_{1} \mathrm{C}_{1} \mathrm{~V}$}

$\mathrm{Au}$ travers des textes étudiés, deux types de réalisation de consonnes latentes (désormais $\mathrm{Cl}$ ) se sont présentés. La première d'entre elles est du type $\mathrm{V} \# \mathrm{ClC}_{1} \mathrm{~V}\left(\right.$ avec $\left.\mathrm{Cl}=\mathrm{C}_{1}\right)$. Prenons les quelques extraits suivants issus de deux textes médiévaux, La passion de Clermont-Ferrand et La chanson de Sainte Foi :

La passion de Clermont-Ferrand:

XI, 43, Palis, vestit, palis, mantenls

davant extendent a'ssos pez.

(« Tissus, vêtements, tissus, manteaux, étendus devant lui, à ses pieds »)

CXVIII, 470, a·ddextris Deu Jesú·se·sset,

( « Jésus s'assis à la droite de Dieu »)

La chanson de Sainte Foi :

XXIII, $234 \quad$ Si-llui non ei, non poiss guerir ;

( Si je ne l'ai pas, je ne puis me sauver »)

XXXIII, 348, a-llui se sun convertid, («à lui ils se sont convertis »)

Ces géminations se retrouvent systématiquement après des monosyllabes tels que les conjonctions :

si (<si) : Sill mog (ChSF, 249), Si-llui non ei, (ibid., 234)

qui (<quid): qui·llo doist (STLeg, 23), Que·tt' asaldran $(P C l, 58)$, que·llor dissest (ibid., 179)

e (<et) : e·lla 'sgarded $(P C l, 50)$, e·llos alquanz $(P C l, 493)$, e' $\mathbf{l l}^{1}$ prometrai $(C h S F, 176)$, e' $\mathbf{l l}^{2}$ homen (ibid., 251), e ss'es ben vera esta razons (ibid., 26), e·sso gran marriment (Boece, 100)

o (<ubi/aut) : oll corps $(C h S F, 357(<u b i))$, o-ss'assis $(P C l, 24(<u b i))$, oll paucs Zacheus $(C h S F$, $530(<a u t))$

Il en est de même après les adverbes :

si (<sic): si-llor dist (STLeg, 206);

no (<non): noll ( $C h S F, 170 ; 173 ; 559)$, no·ssusted $(P C l, 155)$,

Et enfin, des cas de renforcements consonantiques sont également observables après les prépositions $a$ et de:

a (<ad): a·ssos pez ( $P C l, 44)$, a·ssos fedels (ibid., 92), a·nnum li pedre (STAl, 16), a·ssa part (Boece, 105), a llui se sun convertid, (ChSF, 348)

de (<de) : de lla fornaz, (ChSF, 375), de llor cantar, (ibid., 593), de·ssa mort (ibid., 90), de·ssos sanz olz $(P C l, 52)$, de·Il' altra part (ibid., 345)

\subsection{Cas de redoublement syntaxique}

Le raddoppiamento sintattico, ou redoublement syntaxique, est un phénomène d'assimilation complète principalement étudié en italien (Loporcaro, 1997). Il se produit entre deux mots issus d'un même 
syntagme et se caractérise par une gémination de la consonne initiale de Mot2. Il ne se réalise pas de manière identique dans toutes les régions italiennes, et de manière simplifiée, les contextes d'apparition du RS varient principalement entre le toscan et les dialectes centro-méridionaux. Le RS en toscan dépend d'un facteur accentuel, et plus précisément, la consonne initiale du Mot2 sera renforcée si elle est précédée d'un polysyllabe oxyton (città [pp]ulita), ou d'un monosyllabe tonique $(t u$ [dd]ici). L'application de ce RS conditionné accentuellement est tout de même soumise à des restrictions, principalement d'ordre syntaxique. Il doit y avoir un lien sémantico-syntaxique très étroit entre Mot1 et Mot2, on retrouvera le RS uniquement à l'intérieur d'un même syntagme. Ce redoublement synchronique s'explique ainsi phonologiquement, il est régulier, prédictible et même productif, dans la mesure où il se produit également lors d'adaptation d'emprunts. Le RS dans les dialectes centro-méridionaux, quant à lui, se déclenche d'une tout autre manière. Il consiste en un redoublement de type syntaxique, voire morphosyntaxique, celui-ci étant déclenché par une liste fermée de morphèmes. Il s'agit principalement de monosyllabes atones à finale latine consonantique ( $a<a d, e<e t$, no $<$ non, che $<$ quid, plu< $<$ plus...), auxquels s'ajoutent quelques paroxytons (dove, qualche, come) possédant le trait distinctif $[+\mathrm{RS}](d[\mathrm{o}] v e$ [vv]ai). Il faut également souligner l'importance du facteur syntaxique, indispensable pour l'analyse de ce redoublement. En effet, et de la même manière que le redoublement accentuel, les deux mots doivent être très liés syntaxiquement, sans quoi la gémination de la consonne ne s'effectue pas. Le redoublement dans ces dialectes obéit donc à des règles essentiellement syntaxiques, il est irrégulier et est actif en diachronie.

Or si l'on observe les quelques séquences $\mathrm{V} \# \mathrm{C}_{1} \mathrm{C}_{1} \mathrm{~V}$ an ancien français, notées dans la section précédente, ces dernières semblent s'assimiler à ce phénomène de redoublement syntaxique. Tout d'abord, tous les cas de gémination relevés dans les divers textes médiévaux sont représentés par la présence d'un point entre Mot1 et Mot2, et ce détail peut être pertinent dans la mesure où cette graphie ne se présente que rarement dans d'autres contextes. De plus, les géminations à l'initiale de mots, observées dans ces quelques extraits de textes, présentent les mêmes caractéristiques que celles remarquées en italien, et plus précisément dans les dialectes centro-méridionaux. D'une part, on peut remarquer un lien syntaxique très étroit entre le mot présentant un RS et celui qui le précède. Il s'agit principalement de syntagmes prépositionnels ( $a \cdot s \operatorname{sos}$ pez $(P C l, 44)$, « à ses pieds »), adverbiaux (si·llor dist (STLeg, 206), « et lui dit») et quelques conjonctions déclenchent aussi le RS (o.ss'assis $(P C l, 24)$, « où s'asseoir »). Il semble ainsi que les deux mots doivent appartenir à un seul et même syntagme, condition nécessaire, voire indispensable, pour le déclenchement du RS. D'autre part, et encore une fois en accord avec le RS étudié en italien, les mots présentant un redoublement suivent dans presque tous les cas des prépositions ou conjonctions monosyllabiques à finale vocalique, mais qui possédaient une ancienne consonne finale latine $^{3}(a<a d, \text { « à »; si<sic, «ainsi », e<et, « et »,... })^{4}$.

A partir de ces faits, nous analyserons ainsi ces géminations comme des cas de redoublement syntaxique.

\section{Réalisation de la consonne latente sous la forme VCI\#V}

\subsection{Séquences VCI\#V}

Si les anciennes consonnes finales latines réapparaissent dans un contexte de redoublement syntaxique, elles semblent se manifester également dans un contexte différent, du type $\mathrm{VCl} \# \mathrm{~V}$. Plus précisément, une alternance du type [V\#] versus [ $\mathrm{VCl}$ ], selon l'initiale du mot suivant, est observable au travers des textes étudiés, et principalement dans La chanson de Sainte Foi et surtout La vie de Saint Alexis. On retrouve ainsi, dans $S T A l$, plusieurs exemples de ce type :

$e$ vs $e d<e t$ :

XLV, 224, Tut te durai, boens hom, quanque m'as quis,

lit ed ostel e pain e carn e vin.

(« Je te donnerai, saint homme, ce que tu m'as demandé: un lit, (et) un toit, (et) du pain, (et) de la viande et du vin ») 
que vs qued < quid:

LXXVIII, 389, Jo atendi qued a mei reparaisses,

par Deu merci que tum reconfortasses.

(« J'attendis que tu me reviennes, par la miséricorde de Dieu que tu me réconfortes. »)

Ou encore dans $C h S F$ :

$a$ vs $a d<a d$ :

$\begin{array}{ll}\text { XLVII, 543, Fez lor cenbell tot a present ; } & \text { Lo veill tolg ad achella gent, }\end{array}$

(« Il leur livra bataille tout aussitôt, enleva le vieux à ces gens là »)

De manière générale les conjonctions et prépositions présentent toutes des alternances de cette sorte, tout du moins dans le texte STAl. En effet, si l'on observe les formes de la conjonction « et » dans ce poème, les occurrences sous la forme $<\mathrm{e}>$ sont systématiquement en contexte pré-consonantique, alors que devant les mots à initiale vocalique, on retrouve toujours la forme $<\mathrm{ed}>$ :

Occurrences de $<\mathrm{e}>(<e t)$ :

e spiritel, e de la vie, e canter, e de pere, e de mere, e spiritel, e regnet, e suveraine, e dignement, 0 e justise, 2 e fraisles, 9 E Deu, 23 E granz, 105 E sun, 130 e seat, 161 e des regnes, 179 e li petit, $184 \mathbf{E}$ tuit, $185 \mathbf{E}$ de l' honur, 200 e vint, 211 e pur, 223 e pain, e carn, e vin, 225 e le pedre, e le medra, 236 E la pulcele, 237 E de lur, 242 E tut pur, 243 e converset, 261 e set anz, 271 e quatre, 276 e parcamin, 281 e cum, 285 e li rice, e li povre, 302 e li empereör, $306 \mathbf{E}$ tut le, 308 e li empereür, 326 e pensif, e plurus, 327 E ço, 340, 343, $370,380,381$ e cler, e bel, 347 e li empereör, 356 e savie, 375 e de la medre, 379 E cum, 382 E que, 383 E pur, 384 E tantes, 398, 399 e le barbe, 406 e brunie, 411 E grant, 413 e li tons, 415 E d' icels, 418 e sun cors, 427 e sen vis, 428 e debat, 431 E jo, 434 e si, 436 E de ta, 438 E Deus, 454 e goiuse, 458 e de la medre, $466 \mathbf{E}$ tantes, 473 e si pesmes, 480 e le pedra, e la medra, 496 E la pulcela, 497 e bel, 499 E tuit, 508 e li petit, 510 e l' argent, 526 e graciet, $540 \mathrm{E}$ l' anema, 544 e sil servent, 563 e le plus, 564 e de gemmes, 586 e de la medra, $591 \mathrm{E}$ de la spuse, 592 e tuit, 594 E si, 599 e la medra, 601 E la pulcela, 602 e la compaignie, 607 e si boen, 611 e si, 615 e goie, 623

Occurrences de $<\mathrm{ed}>(<e t)$ :

ed es noces, 0 ed amur, 2 ed al tens, 6 Ed al David, 7 ed honurede, 19 ed il fut, 51, Ed un anel, 73 ed enz, 78 ed a la medra, 101 Ed a la, 102 ed a gret, 172 Ed il est, 173 ed ostel, 225 ed enca, 281 Ed une penne, 282 Ed enpur, 404 Ed anpur, 408 ed a, 416 ed acoler, 429 ed eguarede, 470 ed es noces, 516 ed as riches, 537 Ed attement, 569 ed an capes, 582 ed honorethe, 604 Ed en cel, 624

Les mêmes variations s'appliquent également, et ce sans aucune exception ${ }^{5}$, aux conjonctions $<$ se $>/<$ set $>$ $(<$ si + quid), «si », (STAl: se lui, 100 se tei, 202 se Deu, 420, sed il, 128 sed a, 448, etc), $<$ ne $>/<$ ned $>$ $(<n e c)^{6}$, « ni » (Sorz ned avuegles ne contraiz ne leprous, Ne muz ned ors ne nuls palasinous, STAl, 551, 552 ) et $<$ quan $>/<$ quand $>$ (<quando), «quand » (ChSF: quan fo, 112, quan vol morir, 222 quan mor, 324 , quand es, 367 , etc).

La préposition « à » $(<a d)$ présente des changements graphiques elle aussi, (ChSF: a la fin, 3 a celad, 68 a grand tort, 379, ad asserir, 217 ad ella, 445 ad adobar, 505, etc; STLeg : a sos sancz, 2 a flamma, 133 a venir, 212, ad ambes, 119 ad Ostedun, 139, etc)

Enfin, on retrouve les mêmes caractéristiques en ce qui concerne l'adverbe de négation « ne » $(<$ non), qui se réalise sous la forme $<$ ne $>$ en contexte $\# \mathrm{C}$ et $<$ nen $>$ lorsque Mot2 est à initiale vocalique (STAl: ne cesset, 85 ne luin, 92 ne puis, 110, nen at parfite amour, 68 nen est merveille, 465 nen out si grant, 536, etc). 
Cette alternance graphique est ainsi sans exception à travers ces textes. Précisément, elle concerne tous les monosyllabes atones dans $S T A l$, et seuls quelques uns d'entres eux dans les autres textes mais toujours de manière systématique ${ }^{7}$. Or cette variation du type $[\mathrm{V \# C}]$ versus $[\mathrm{VCl} \# \mathrm{~V}]$ semble pouvoir s'assimiler au phénomène de liaison du français moderne.

\subsection{Cas de liaison}

La liaison est un type de sandhi externe propre au français moderne, qui consiste en la prononciation de la consonne finale de Mot1, muette dans le mot pris isolément, lorsque Mot2 est à initiale vocalique. Ainsi, si le syntagme "grand garçon » se dit [grã-garsõ], il est par contre indispensable de prononcer "grand ([grã]) enfant ([ãfã]) » tel que [grã-t-ããa ] et non [grã-ãfã].

La condition nécessaire à la liaison est le lien syntaxique, voire sémantico-syntaxique, qui doit impérativement exister entre Mot1 et Mot2 de manière très étroite ${ }^{8}$. Ainsi si l'on prend l'exemple de la phrase « le petit arbitre la rencontre demain », on peut remarquer une ambiguité. Cette séquence peut être découpée syntaxiquement de deux sortes :

\section{Soit (1) [le petit | arbitre la rencontre demain] soit (2) [le petit arbitre | la rencontre demain].}

Dans la première découpe, le syntagme nominal contient uniquement le nom " petit », alors que dans la seconde il est composé du nom « arbitre » et de l'adjectif «petit». Par conséquent, on prononcera (1) par [ləpətiarbitr...], « petit » et « arbitre » étant issus de deux syntagmes distincts, contrairement à (2) qui se produit $[\operatorname{lop}(\partial)$ titarbitr...] avec réalisation de la consonne latente, "petit » et "arbitre » étant très liés syntaxiquement. De la même manière, si l'on prend la cas d'un adjectif à variation finale tel que « nouveau », on obtiendra, en accord avec ce qui vient d'être dit, les phrases suivantes : (1) Le nouveau arbitre la rencontre demain et (2) Le nouvel arbitre la rencontre demain.

Si l'on observe les séquences notées en 3.1, des similitudes avec le phénomène de liaison semblent repérables en ancien français. Dans un premier temps, la variation est identique au français, à savoir une alternance de séquences V\#C (e justise, $S T A L, 2$; « grand garçon » ([grãgarsõ])) contre VC\#V (ed amur, ibid.; "grand enfant » ([grãtãfã])). De plus, et de la même manière qu'en français, il existe un lien sémantico-syntaxique très étroit entre Mot1 et Mot2. Les cas de liaison en ancien français se produisent en effet systématiquement après des monosyllabes atones de type adverbial, prépositionnel ou conjonctionnel :

et Amazonas e Pigmeu, "et les Amazones et les Pygmées » (ChSF, 489), ned avuegles ne contraiz (STAl, 551), Fez lor cenbell tot a present; Lo veill tolg ad achella gent, " il leur livra bataille tout aussitôt, enleva le vieux à ces gens là » $(C h S F, 543,544)$, etc.

Or s'il existe des liaisons de type facultatif en français, celles entre un clitique et son support sont, quant à elles, obligatoires. Et c'est pourquoi, dans une séquence « déterminant + nom » respectant les conditions phonologiques de la liaison, le phénomène s'appliquera alors sans exception : "les enfants », de même que « un enfant » contiennent respectivement les consonne de liaison [z] et [n] ([lezãfã], [ẽnãfã]). Les variations relevées en ancien français sont donc en accord avec le phénomène de liaison, en ce qui concerne le rapport syntaxique entretenu entre les deux mots.

Par contre, une différence peut être constatée entre le français et l'ancien français à propos de la catégorie grammaticale de Mot1. En effet, si les cas de liaison relevés dans les textes médiévaux touchent principalement les conjonctions et prépositions, seules très peu d'entre elles sont concernées par ce phénomène en français moderne. Et pour cause, elles ne présentent pas de consonne finale latente pour la plupart: «si, ne, ni, que, à ». On retrouve dans «et» une consonne graphique mais qui n'est pas considérée comme latente. Cette conjonction bloque en effet la liaison, et fait partie des quelques cas de liaisons dites « interdites ». En ce qui concerne « quand», les règles de liaison s'applique lorsque le mot suivant est à initiale vocalique («quand il arrivera...»: [kãtilarivra]). Et inversement, si en français beaucoup de liaisons sont présentes après les adjectifs pré-nominaux, ce n’est pas le cas en ancien français, tout du moins à la période étudiée. 


\section{La latence en ancien français ( $\mathrm{X}^{\mathrm{ème}}$ et $\mathrm{Xl}^{\mathrm{ème}}$ siècles)}

Dans les parties précédentes, nous venons d'observer la présence de consonnes à la finale de clitiques, normalement absentes dans le mot isolé, aussi bien en contexte \# $\mathrm{V}$ qu'en contexte \# \#. Les mots déclenchant ce phénomène étant à finale latine consonantique (voir note 4 ), l'idée est donc qu'à la chute de ces consonnes finales, ces dernières ont laissé une trace, créant ainsi une consonne latente, c'est-à-dire une consonne muette mais lexicalisée, qui réapparaît sous certaines conditions syntaxiques (Mot1 et Mot2 doivent être très liés). Je vais ainsi tenter de vérifier cet éventuel statut de latence en analysant ces consonnes finales, en contexte pré-vocalique puis pré-consonantique.

\subsection{Analyse de la liaison}

Les cas de liaison vus en 3 . semblent pouvoir s'analyser de manière identique à la liaison propre au français moderne. La consonne de liaison, absente en contexte _ $\# \mathrm{C}$, est systématiquement réalisée en contexte _ \# V lorsque Mot1 entretient une relation syntaxique très étroite avec Mot2.

Le traitement de ces consonnes en ancien français semble donc similaire à celui en français moderne, et peut ainsi s'ancrer dans les diverses théories qui ont été formulées ces dernières années. Pour n'en citer qu'une, Sauzet (1999) a émis une hypothèse basée sur une approche bien spécifique de la « linéarité », consistant à dire que la linéarité serait «créée » par la syllabation, la successivité dans les morphèmes et entre les morphèmes, dépendant de celle-ci. Je ne m'attarderai pas ici sur cette notion complexe et pour résumer très brièvement, le principe de la théorie de Sauzet est de dire que la syllabation procède de droite à gauche, selon un mécanisme bien précis, basé sur une syllabe [((attaque) (noyau (coda)))] de type $X^{\prime},[(($ adjonction) (tête (complément)))]. Selon lui, les consonnes latentes doivent être lexicalisées tout en ayant un statut particulier. Il soumet alors l'idée de consonnes non associées au reste du contenu phonique, c'est-à-dire qu'elles existent réellement au niveau morphémique, mais de manière discontinue. Il est ainsi question de consonnes «dissociées». Sauzet propose ainsi de représenter les mots à consonnes latentes, comme «petit» de la manière suivante :

$$
\text { «petit » } \rightarrow \text { p-ə-t-i- } \boldsymbol{A} \boldsymbol{d} \boldsymbol{j} \text {-t }
$$

Pareillement, je note donc la préposition «à » en ancien français/a-d/, avec une Cl qui appartient lexicalement au mot mais qui sera linéarisée indépendamment de /a/. Le segment suivant pourra alors légitimer ou non la consonne latente, ou dissociée (qui formera alors une attaque), selon que ce segment sera identifié ou non comme tête (ou noyau). Dans le syntagme /a-d $+\varepsilon l a /, / \varepsilon /$ est identifié comme tête et peut donc se lier soit à /a/ soit à /d/. La consonne dissociée sera préférée car elle permet de former une attaque au contraire de [a], on obtiendra ainsi le syntagme [adela].

Pour résumer, il semble convaincant d'admettre que la chute des consonnes finales latines a créé un contexte de latence, qui s'est manifesté dès l'ancien français par l'apparition de ces consonnes latentes devant les phonèmes vocaliques, lorsque les deux mots entretiennent un rapport syntaxique étroit.

D'ailleurs, en ce qui concerne ce lien syntaxique, celui-ci est finalement toujours présent entre Mot1 et Mot2. En effet, parmi les données étudiées, on ne trouve aucun cas où Mot1 n'est pas lié au mot suivant syntaxiquement, ce qui entraînerait alors la non réalisation de la $\mathrm{Cl}$. Mais ceci n'est pas inattendu si l'on observe le type de mots porteurs de $\mathrm{Cl}$. Effectivement, nous avons vu que seules les conjonctions et prépositions contiennent des $\mathrm{Cl}$ et celles-ci ne peuvent être utilisées en fin de syntagme (ce qui bloquerait la réalisation de la $\mathrm{Cl}$ ) dans la mesure où elles doivent être liées à leur support.

Et le fait que les liaisons ne touchent aucune autre catégorie grammaticale, tels que les adjectifs prénominaux, n'est finalement pas étonnant au regard de l'évolution des consonnes finales latines. Plus précisément et de manière très simplifiée, les $\mathrm{Cl}$ ont pour particularité d'être muettes dans le mot isolé mais une étude diachronique de ces consonnes montre qu'elles étaient toutes prononcées en latin. Un phénomène d'effacement consonantique s'est produit entre les I ${ }^{\text {er }}$ et III ${ }^{\text {ème }}$ siècles, et les consonnes finales latines se sont alors amuïes ( $\operatorname{sic}>$ si, nec $>n e, a d>a \ldots$ ), excepté les finales $/ \mathrm{t} / \mathrm{et} / \mathrm{s} /$, conservées plus longtemps en raison de leur fonction verbale flexionnelle (cantat (« il chante ») vs cantas (« tu chantes »). 
Les mots à finale vocalique quant à eux, dont font partie les adjectifs, ont connu dans un premier temps la chute de la voyelle finale ${ }^{10}$ aux VII ${ }^{\text {ème }}$ et VIII ${ }^{\text {ème }}$ siècles (grande $>$ grand). La consonne désormais en position finale, dite «consonne finale romane ", commencera à s'amuïr seulement au XII ${ }^{\text {ème }}$ siècle, après avoir subit un phénomène de dévoisement. L'évolution de l'adjectif « grand » est ainsi la suivante :

$$
\operatorname{grand} \underline{\operatorname{eg}}>\operatorname{grand}\left(\mathrm{VII}{ }^{\mathrm{ème}}\right)>\operatorname{grant}\left(\mathrm{VIII}^{\mathrm{ème}}\right)>\operatorname{gran}\left(\mathrm{XII}^{\mathrm{ème}}\right)
$$

Les textes étudiés datant des $\mathrm{X}^{\text {ème }}$ et $\mathrm{XI}^{\text {ème }}$ siècles, il n'est donc pas surprenant de relever des cas de liaison uniquement en finale de conjonctions, prépositions, et adverbes dans la mesure où seuls ces derniers comportent des consonnes finales latines qui se sont amuïes plusieurs siècles avant la période étudiée, contrairement aux finales d'adjectifs. De la même manière, il n'est pas surprenant que les pronoms soient exclus de cette discussion puisque tout comme les adjectifs, ceux-ci étaient à finale vocalique (je, me $<e g o, m e i$; tu, toi $<t u$, te; il, lui $<i l l e$, illi). Seules les formes plurielles avaient une finale consonantique $-s$, mais cette dernière a été conservée en ancien français pour sa fonction flexionnelle (nos, vos (latin) > nos/nous, vos/vous (AF)).

\subsection{Analyse du redoublement syntaxique}

\subsubsection{Le RS : hypothèse d'une syllabe à noyau nul}

En ce qui concerne le RS, les mêmes mots impliqués dans le phénomène de liaison déclenchent le renforcement consonantique à l'initiale de Mot2, il semble donc on ne peut plus logique de faire une hypothèse diachronique quant à la réalisation du RS, en supposant que les consonnes finales latines sont également liées à ces géminations. En partant de ce postulat, une description phonologique synchronique semble tout à fait appropriée, basée sur l'idée que si les consonnes finales latines se sont amuïes, leurs positions, quant à elles, n'ont pas pour autant disparu. Une telle perspective repose ainsi sur le concept de latence, au même titre que les consonnes de liaison. Nous ne tiendrons pas compte dans un premier temps des deux mots déclencheurs à finale vocalique de et si (note 4), mais uniquement de ceux qui déclenchent également la liaison. Nous verrons seulement par la suite s'ils peuvent ou non remettre en cause la théorie alors énoncée.

Pour tenter d'étudier ce phénomène de renforcement consonantique, je vais m'appuyer sur la théorie de Sauzet vue dans la section précédente, c'est-à-dire celle qui s'inscrit dans le cadre de la latence analysée comme dissociation, ainsi que sur son analyse des consonnes finales occitanes (2004) qu'il traite en se basant sur le principe de la syllabe à noyau nul (CØ). Cette notion a été proposée par Dell (1995) pour légitimer les consonnes finales dites «excédentaires». Plus précisément, partant du postulat que les syllabes finales de mot doivent se terminer par une voyelle, les consonnes finales qui ne sont pas suivies d'un segment vocalique excèdent le format d'une syllabe régulière et nécessitent ainsi un mécanisme de légitimation spécifique. Dell (1995) soumet l'idée d'une (CØ), dans laquelle la coda en excès devient alors attaque du noyau nul.

Partons donc du principe que la consonne latente en ancien français consiste en une syllabe à noyau nul, le mot «à » ayant ainsi comme représentation $[\mathrm{a}(\mathrm{C} \varnothing)]$ ou même $[\mathrm{a}(\mathrm{d} \varnothing)]$ (une même analyse a déjà été effectuée par Russo \& Giuliani (2004), en ce qui concerne le RS « en latin tardif et médiéval »). La légitimation de cette consonne s'effectuerait alors selon un principe accentuel dans un premier temps. Plus précisément, dans les langues occitanes (Sauzet, 2004), l'accent de mot entraîne la réalisation de celle-ci. Cependant, si le mot contenant la (CØ) se trouve très lié syntaxiquement au mot suivant, alors l'accent de Mot1 s'efface en faveur de l'accent de syntagme. La consonne finale (ou attaque de (CØ)) n'est ainsi plus légitimée par l'accent et tend à s'effacer. La syllabation s'effectuant de droite à gauche, le morphème suivant pourra alors conserver le noyau de la syllabe finale en le rattachant à l'attaque de Mot2. C'est ainsi que Russo \& Giuliani expliquent les cas de gémination en latin tardif, et de la même manière, en ancien français, on suppose que dans la suite «à + ses pieds», a.ssos pez $(P C l, 44)$, l'ancienne consonne finale [d] de $a$ n'étant pas légitimée par l'accent ( $a$ est un morphème atone), la coda /d/ s'efface, mais la syllabe à noyau nul peut être maintenue dans la mesure où le pronom sos entretient 
des relations syntaxiques fortes avec le morphème $a$, et celle-ci sera alors légitimée en se liant à l'attaque du mot suivant, d'où la gémination de l'attaque initiale de Mo2 :

$$
a \cdot \operatorname{ssos}(\text { pez }): /(\mathrm{a}(\mathrm{d} \varnothing))(\mathrm{s} \supset \mathrm{s}) / \rightarrow[\mathrm{a} \# \mathrm{ss} \mathrm{s}]
$$

\subsubsection{Le RS : phénomène accentuel}

Les analyses précédentes semblent ainsi apporter une explication unitaire quant à la latence en ancien français, à savoir que les consonnes latentes, consistant en une ancienne finale latine, se manifestent aussi bien en contexte pré-consonantique que pré-vocalique quand Mot1 et Mot2 sont liés syntaxiquement. Cependant, il existe quelques lacunes à cette supposition, et principalement en ce qui concerne la réalisation du $\mathrm{RS}$.

Si la théorie basée sur la présence d'une syllabe à noyau nul permet d'expliquer les cas d'assimilation dans les langues occitanes, quelques problèmes se posent en revanche en ancien français. Entre autres, on s'attend à trouver un nombre plus important de RS. Prenons le cas de $a$ dans $C h S F$, très peu de RS sont relevés, or on s'attend, d'après l'hypothèse qui vient d'être énoncée, à en voir nettement plus. Plus précisément, au regard de tous les cas de «non-liaison », la (CØ) devrait être liée à l'attaque de Mot2 de la même manière que dans $a \cdot s s o s$. Ainsi, a sun (parent) (« à son père », ChSF, 548), devrait connaître une gémination $\left([(\mathrm{a}(\mathrm{d} Ø)) \operatorname{sun}] \rightarrow^{*}[\mathrm{a} \#\right.$ ssun $\left.]\right)$.

Dans les langues occitanes, et précisément en languedocien (Sauzet, 2004), l'accent de mot peut légitimer la (CØ), par conséquent ne serait-ce finalement pas un phénomène accentuel qui produirait la gémination en ancien français? Les propriétés accentuelles de l'ancien français diffèrent de celles du français étant donné la présence d'accents toniques de mot, et non de syntagme, ainsi que le principe de l'accent secondaire qui était encore actif (Marchello-Nizia, 1999). A partir de ce constat, et sachant que les mots déclencheurs de RS et liaison sont atones, on peut supposer qu'en revanche certains possèdent un accent secondaire qui, au même titre que l'accent primaire, engendre la réalisation de la $\mathrm{Cl}$. Et d'après une analyse prosodique du corpus de RS, les mots déclencheurs semblent en effet pouvoir recevoir un accent secondaire si l'on s'en tient aux règles de Vogel \& Scalise (1982), émises en ce qui concerne l'italien (1. accentuer de préférence la première syllabe d'une séquence. 2. procéder en alternant syllabe accentuée/syllabe non accentuée. 3. ne pas laisser deux syllabes contiguës non accentuées). Les exemples suivants illustrent ainsi l'accentuation ${ }^{11}$ possible des syntagmes présentant un RS :

\begin{tabular}{|c|c|c|}
\hline a'ssos pez & $\rightarrow$ & [à-ssวs-pźz] ( «à ses pieds », $P C l, 44)$ \\
\hline $\boldsymbol{o} \cdot \mathbf{s s} \mathbf{\prime}^{\prime}$ ssis & $\rightarrow$ & [ò-ssa-sís] (« où s'assoir », ibid., 24) \\
\hline si.llor dist & $\rightarrow$ & [sì-llor-díst] («et lui dit », STLeg, 206) \\
\hline $\boldsymbol{e} \cdot \mathbf{s s}$ gran (marriment) & $\rightarrow$ & [è-sso-grá] (« et sa grande douleur », Boece, 100) \\
\hline no:ssusted & $\rightarrow$ & [nò-ssus-tźd] (« il ne resista pas », $P C l, 155)$ \\
\hline
\end{tabular}

Cette approche accentuelle permet de rendre compte de la présence ou de l'absence d'un RS après un même mot déclencheur dans un contexte syntaxique similaire, le principe étant que selon l'accentuation ou la non accentuation d'un mot, il légitime alors ou non la $\mathrm{Cl}$, d'où la production d'une assimilation :

$$
/(\mathrm{a}(\mathrm{Cl}))(\mathrm{s} \supset \mathrm{s}) / \quad \rightarrow \quad[\mathrm{a} \# \mathrm{ss} \supset \mathrm{s}]
$$

Dans ce syntagme, la consonne latente est légitimée par l'accent du noyau [à], la $\mathrm{Cl}$ est ainsi réalisée sous la forme d'une assimilation complète avec le segment suivant [s] 


\subsection{La latence en ancien français : résumé et lacunes}

Pour résumer tout ce qui vient d'être dit, observons les différentes réalisations de la $\mathrm{Cl}$ après la préposition $a$ :

$$
\begin{aligned}
& \text { a Deu } \quad: \quad[(\mathrm{a}(\mathrm{Cl}))(\mathrm{deu})] \quad \rightarrow \quad[\mathrm{a} \# \mathrm{deu}] \\
& \text { ad ella : } \quad[(\mathrm{a}(\mathrm{Cl}))(\text { cla })] \quad \rightarrow \quad \text { [a\#dela] }
\end{aligned}
$$

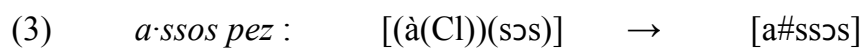

On pose donc en coda du monosyllabe une consonne latente. On avance l'hypothèse que l'accentuation de la syllabe contenant la $\mathrm{Cl}$ entraîne sa réalisation. Les mots à finale consonantique latente sont tous des morphèmes atones, on présuppose alors qu'ils peuvent recevoir un accent secondaire qui, dès lors, légitimera la $\mathrm{Cl}$ qui sera rattachée, par assimilation complète, à l'attaque du mot suivant (exemple (3)). Lorsque Mot1 n'est pas accentué, la légitimation de la $\mathrm{Cl}$ ne pourra se faire que syllabiquement. Partant du principe que la syllabation est récursive et se produit de droite à gauche, c'est donc le segment initial de Mot2 qui autorisera ou non la réalisation de la $\mathrm{Cl}$. Si ce segment est identifié comme tête, il légitime la $\mathrm{Cl}$ qui devient alors attaque de ce noyau (2), dans le cas contraire, la $\mathrm{Cl}$ s'efface (1).

Les consonnes latentes en ancien français semblent ainsi avoir la même origine, à savoir la chute des consonnes finales en latin, et se réalisent aussi bien en contexte _ \# V (liaison) que _ \# C (RS), selon des principes respectivement syllabique et accentuel.

Ceci étant, un élément, jusqu'ici laissé de côté, est susceptible de remettre en considération cette hypothèse. Il s'agit de la présence de RS après de et si. Et pour cause, ces morphèmes ne présentaient pas de consonne finale en latin $(<d e ; s i)$. Or de la même manière qu'il n'y a pas de cas de liaison après de et si en contexte _ \# V , la présence du RS après ces morphèmes reste inattendue au regard de la théorie émise jusqu'ici, basée sur la présence de consonnes latentes correspondant aux finales latines. Comment expliquer alors la gémination dans les syntagmes de ssa mort (" de sa mort », ChSF, 90), de lla fornaz ( «de la fournaise », ibid., 374), de Ssos sanz olz (« de ses yeux saints», PCl, 52), Sill mog («s'il lui porta », ChSF, 249), Si llui non ei, («si je ne l'ai pas», ibid., 234)? Faute de recherches encore suffisamment approfondies, nous ne donnerons pas de solution définitive à cette question au terme de cette discussion, mais uniquement les possibilités envisagées.

Le même problème se pose en italien et plusieurs auteurs (Absalom \& Hajek, 1997 ; Loporcaro, 1997) expliquent ces cas en admettant qu'ils ont été ajoutés par analogie. L'argument de l'analogie peut sembler concluant dans le contexte de l'ancien français, dans la mesure où elle se produit à l'intérieur d'un même paradigme morphologique. Aucun mot ne provoque de RS hormis de et $s i$, or ces derniers sont des adverbe et conjonction monosyllabiques, pareillement aux mots déclencheurs de RS à finale latine consonantique.

Si de et si ont été ajoutés par analogie, alors les cas de RS après ces morphèmes devraient commencer à apparaître plus tard que ceux déclenchés par les mots à finale latine consonantique. Les textes étudiés ici ne révèlent aucun détail chronologique quant à ces mots déclencheurs, mais les premiers cas de RS datant a priori du latin vulgaire (Russo \& Giulani, 2004) une étude diachronique permettra de vérifier ce fait.

Un bémol doit tout de même être ajouté par rapport à cette supposition. S'il était question d'analogie, alors ce devrait être par soucis de simplification grammaticale. Or les consonnes géminées ayant disparu au VII ${ }^{\text {ème }}$ siècle, le RS relevé dans ces textes du $\mathrm{X}^{\text {ème }}$ et $\mathrm{XI}^{\text {ème }}$ siècles est donc considéré comme un phénomène marqué. Par conséquent, l'analogie reste une hypothèse acceptable s'il existait déjà des cas de RS après de et $s i$ avant le VII ${ }^{\text {ème }}$ siècle. Tout ceci devra être confirmé lors de recherches ultérieures.

Mais quoi qu'il en soit, si la présence de RS après de et si peut remettre en cause l'idée de la latence en contexte _ \# C, elle confirme en revanche l'idée du rôle accentuel dans la réalisation des géminations. En effet, en partant du principe que les mots déclencheurs de RS ne possèdent pas de consonne latente, l'hypothèse accentuelle avancée précédemment reste la plus probable. Dans ce cas, le RS consisterait 
alors uniquement en un phénomène accentuel, et serait en définitive totalement distinct du phénomène de liaison. La règle suivante rend compte d'un tel principe :

$$
\mathrm{C} \rightarrow \mathrm{CC} / \quad\left[\mathrm{V}[+\mathrm{acc}] \#_{-}\right]
$$

A partir de cette règle, tous les cas de RS s'expliquent aisément. Si l'on reprend le cas de $a \cdot s s o s$ pez tout comme de ssa mort, si Mot1 possède un accent secondaire, alors la consonne initiale de Mot2 sera géminée.

\section{Conclusion}

Pour conclure, il semble donc tentant d'envisager la présence, en ancien français, d'un même phénomène de latence se réalisant aussi bien en contexte _ \#V qu'en contexte _ \#C, au regard des mots déclencheurs et du contexte syntaxique similaires. L'idée étant que Mot1 peut légitimer accentuellement la $\mathrm{Cl}$ qui sera alors complètement assimilée à l'attaque de Mot2, et si tel n'est pas le cas la syllabation du syntagme produira ou non la $\mathrm{Cl}$, selon que le segment suivant soit identifié ou non comme tête (segment vocalique).

Ceci étant, cette analyse reste valide à l'unique condition de considérer les cas de renforcement consonantique après la préposition de et la conjonction si comme des analogies. Dans le cas contraire, le RS consisterait en un phénomène purement accentuel, aucunement lié à un principe de latence consonantique. Des recherches ultérieures seront menées afin de vérifier ce fait. Mais quoi qu'il en soit, que le RS constitue ou non un résidu de l'ancienne consonne finale latine, l'hypothèse spécifiant qu'il se réalise selon un principe accentuel reste assumée.

Il reste également à terminer l'étude approfondie du type de consonnes géminées, qui pourrait apporter de nouveaux éléments, susceptibles d'être pertinents dans l'étude de ce RS. D'ailleurs, les prémisses de cette analyse montrent que la consonne géminée la plus présente parmi les sept relevées est /1/ (si.llor, a llui, qui $\cdot l l o, e \cdot l l a \ldots)$. Or au-delà de l'éventuel intérêt de cette précision pour l'étude du RS en ancien français, cette dernière observation pourrait également permettre de mieux comprendre l'existence de cette même gémination [11] en français moderne. Effectivement, si les consonnes doubles sont très rares en français, il existe tout de même quelques cas tels que «immoral », «illisible », « surréaliste », etc. Et un autre cas de gémination est entendu lors de l'utilisation du pronom objet à la troisième personne. Prenons les phrases, « la maison qu'il (a) construit(e)» (1) et "c'est lui qui l'a construite » (passé) ou « c'est lui qui la construit» (présent) (2), on remarque une gémination de la liquide /l/ dans «l'a » tout comme «la » en (2) :
(1) la maison [kila] constuite
(2) c'est lui [kil:a] construit(e)

Carvalho (2002) a avancé une théorie synchronique de cette gémination, en posant /11/ comme signifiant du pronom objet, mais au regard des géminées principalement représentées par $/ 1 /([11])$ aux $\mathrm{X}^{\text {ème }}$ et $\mathrm{XI}^{\text {ème }}$ siècles, l'étude des consonnes doubles envisagée permettra peut-être de proposer une explication diachronique à ce renforcement consonantique présent en français.

\section{Références}

Absalom, M. \& Hajek, J. (1997), Raddoppiamento sintattico: what happens when the theory is on too tight?, in P.M. Bertinetto et al. (eds) Certamen Phonologicum III, Rosenberg \& Sellier, pp. 159-179

Avalle, d'A. S. (2002), La doppia verità, Fenomenologica ecdotica e lingua letteraria del medioevo romanza, Galluzzo.

Carvalho, J. (2002). De la syllabation en termes de contours CV. Mémoire accompagnant la demande d'Habilitation à diriger des recherches, École des Hautes Etudes en Sciences Sociales.

Encrevé, P. (1988), La Liaison avec et sans enchaînement. Phonologie tridimensionnelle et usages du français. Paris : Seuil. 
Fougeron C., Goldman J.P., Dart A., Guélat L. \& Jeager C. (2001), Influence de facteurs stylistiques, syntaxiques et lexicaux sur la réalisation de la liaison en français. 8ème Conférence Traitement Automatique des Langues Naturelles, Tours, France.

Lavaud, R. \& Machicot, G. (1950), Boécis, poème sur Boèce, Toulouse, Institut d'Etudes Occitanes.

Linskill, J., (1974), Saint-Léger. Étude de la langue du manuscrit de Clermont-Ferrand, suivie d'une édition critique du texte, Genève.

Loporcaro, M. (1997), L'origine del raddoppiamento fonosintattico. Saggio di fonologia diacronica romanza, Basel und Tübingen : Francke verlag (Romanica Helvetica vol. 115).

Marchello-Nizia, C. (1999), Le français en diachronie : douze siècles d'évolution, Paris-Gap, Ophrys.

Perugi, M. (2000), La vie de Saint Alexis, Droz.

Paris, G. (1933), La vie de saint Alexis : poème du XIe siècle / texte critique de Gaston Paris. Septième édition Revue. Les classiques français du Moyen âge. Paris : Champion.

Paris, G. \& Pannier, L. (1872), La vie de saint Alexis, poème du XI siècle et renouvellements des XII ${ }^{e}$ XIII ${ }^{e}$ et XIV siècles publiés avec préface, variantes, notes et glossaire par Gaston Paris et Léopold Pannier. Bibliothèque de l'École des hautes études. Sciences historiques et philologiques, 7. Paris : Franck.

Russo, M. \& Giuliani M. (2004), Redoublement syntaxique (RS) et consonnes latentes en latin tardif et médieval: quelques repères, Actes des JEL 2004, Crouzet, O., Darmidache, H., Wauquier, S. (eds.), a.a.i. (JE2220 acoustique, acquisition, interprétation), UFR Lettres et Langage, Université de Nantes, p. 109-118.

Sauzet, P. (1999), Linéarité et consonnes latentes, Recherches linguistiques de Vincennes, 28, p. 59-86.

Sauzet, P. (2004), Variation des finales occitanes et format de la syllabe, Nouveaux départs en phonologie : les conceptions sub- et suprasegmentales. T. Meisenburg \& M. Selig eds. Tübingen : Gunter Narr, p.33-48.

Storey, C., (1968), La vie de Saint Alexis, Droz.

Thomas, A. (1974), La chanson de Sainte Foi d'Agen, Paris, Champion.

Vogel, I. \& Scalise, S. (1982), Secondary Stress in Italian, Lingua 58, pp. 213-242

\footnotetext{
${ }^{1}$ Ici $<$ ll $>$ correspond au pronom objet « lui ».

${ }^{2}$ Ici $<$ ll $>$ correspond au déterminant pluriel « les ».

${ }^{3}$ La plupart des consonnes finales ont connu un phénomène d'effacement qui s'est produit entre les Ier et IIIème
} siècles.

${ }^{4}$ Seulement deux mots déclencheurs de RS étaient à finale latine vocalique, la préposition de ( de ») et la conjonction si («si », et non l'adverbe si, « ainsi », issu de sic). Nous traiterons dans un premier temps les cas de RS en faisant abstraction de ces derniers, pour ne prendre en compte que les mots déclencheurs à la fois de RS et de liaison.

${ }^{5}$ En ce qui concerne que (<quid) dans $S T A l$, on peut lire au vers 456 quet vedisse, mais il ne s'agit pas ici d'une exception, quet provenant ici de la suite que + te (« (avant) que je te vois »).

${ }^{6}$ Je note ici le cas de ne/ned $(<\mathrm{nec})$, conformément à la version de Paris (1933). L'auteur explique ce [d] issu d'un [k] par analogie avec d'autres mots présentant des cas de liaison: "J'ai distingué [...] le $\mathrm{d}$ et le $\mathrm{t}$ caducs, du $\mathrm{d}$ et du $\mathrm{t}$ stables en les marquant d'un point souscrit ([noté ici par une apostrophe]). Le $d$ ne paraît qu'à l'intérieur des mots, le $t$ qu'à la fin. [...] J'ai seulement écrit, pour les représentants d'ad, et, aḍ, eḍ devant voyelle, $a, e$ devant consonne ; de même qued, seḍ, neḍ (formes analogiques) et que, se, ne [...]» (p.IV). En revanche, dans la version antérieure (Paris, Pannier, 1872), le $<\mathrm{d}>$ n'apparaît pas à la finale de $n e$ en contexte \#V.

${ }^{7}$ Dans $C h S F$, on retrouve des cas de liaison uniquement après $a$ et $e$, mais les autres monosyllabes présentent le phénomène d'élision ( $q$ 'ell, 105, s'ad ella, 445, n'altra, 584). 


\footnotetext{
${ }^{8} \mathrm{Si}$ je ne cite dans cette étude que l'importance du contexte syntaxique, d'autres facteurs (prosodiques, stylistiques, phonétiques...) doivent également être pris en considération dans l'étude de la réalisation de la liaison (Fougeron et al., 2001).

9 Je note $\mathrm{VC \# V}$ en accord avec la graphie, mais au niveau phonologique il serait évidemment plus juste de noter $\mathrm{V \# CV}$ puisque la consonne de liaison est considéré en général comme attaque de Mot2 (dans sa réalisation la plus courante qu'est la liaison avec enchaînement).

${ }^{10}$ Excepté certains cas dans lesquels les voyelles finales latines se sont simplement affaiblies et correspondent actuellement à un schwa : -a (porta>porte); $-\mathrm{CCV}$ avec $\mathrm{CC}=/ \mathrm{C}+1, \mathrm{r}, \mathrm{m}, \mathrm{n} / \mathrm{ou} / \mathrm{C}$ labiale $+\mathrm{y} /$ (patre $>$ père); dans les proparoxytons (cómite $>$ comte)

${ }^{11}$ J'ai placé l'accent primaire (') et secondaire (') sur le noyau de la syllabe accentuée.
} 